\title{
Supersymmetric unparticle effects on Higgs boson mass and dark matter
}

\author{
N.G. Deshpande ${ }^{\mathrm{a}, *}$, Xiao-Gang He ${ }^{\mathrm{b}}$, Jing Jiang ${ }^{\mathrm{a}}$ \\ ${ }^{a}$ Institute of Theoretical Science, University of Oregon, Eugene, OR 97403, USA \\ ${ }^{\mathrm{b}}$ Department of Physics and Center for Theoretical Sciences, National Taiwan University, Taipei, Taiwan
}

Received 3 August 2007; accepted 17 September 2007

Available online 21 September 2007

Editor: M. Cvetič

\begin{abstract}
We propose a model that introduces a supersymmetric unparticle operator in the minimal supersymmetric Standard Model. We analyze the lowest dimension operator involving an unparticle. This operator behaves as a Standard Model gauge singlet and it introduces a new parameter into the Higgs potential which can provide an alternative way to relax the upper limit on the lightest Higgs boson mass. This operator also introduces several unparticle interactions which can induce a neutral Higgsino to decay into a spinor unparticle. It also induces violation of scale invariance around the electroweak scale. It is necessary for the scale of this violation to be larger than the lightest supersymmetric particle mass to maintain the latter as the usual weakly interacting massive particle dark matter candidate. An alternative is to have unparticle state as dark matter candidate. We also comment on some collider implications.
\end{abstract}

(c) 2007 Elsevier B.V. All rights reserved.

In a scale invariant theory in four space-time dimensions, the mass spectrum of fields is zero or continuous. Since in the real world there are plenty of particles with discrete non-zero masses, scale invariance, had it existed, must have been broken at some high energy scale beyond the Standard Model (SM) scale. At high energy there may be both a scale invariant sector and a sector which does not obey scale invariance, such as the SM fields. Recently Georgi proposed an interesting idea to describe possible scale invariant effects at low energies with an operator $O_{\mathcal{U}}$, termed unparticle [1], which interacts with the operator $O_{\mathrm{SM}}$ composed of SM particles. At certain scale $\Lambda_{\mathcal{U}}$ the scale invariant sector with infrared fixed point induces dimensional transmutation, and below that scale operators $O_{\mathrm{SI}}$ composed of scale invariant fields matches on to an unparticle operator $O_{\mathcal{U}}$ with dimension $d_{\mathcal{U}}$. The unparticle interaction with SM particles at low energy has the form

$$
\lambda \Lambda_{\mathcal{U}}^{4-d_{\mathrm{SM}}-d_{\mathcal{U}}} O_{\mathrm{SM}} O_{\mathcal{U}}
$$

The unparticle idea has received a lot of attention [1-4]. In Ref. [4] a class of operators involving SM particles and unparticles are listed. Using these operators one can study unparticle phenomenology in a systematic way. There have been extensive studies of unparticle effects at low energies, which can be used to constrain the unparticle physics scale.

When the scale invariant sector has interactions with the SM sector, the scale invariance will be broken. For example an interaction of the form $\lambda \Lambda^{2-d_{\mathcal{U}}} H^{\dagger} H O_{\mathcal{U}}$ will break scale invariance, after the Higgs field develops a vacuum expectation value $v / \sqrt{2}$, since a term of the form $\lambda \Lambda^{2-d \mathcal{U}} v^{2} O_{\mathcal{U}}$ will be generated. A Yukawa type of coupling $\lambda \Lambda^{-d_{\mathcal{U}}} \bar{Q}_{L} H U_{R} O_{\mathcal{U}}$, at one loop level can generate a term of the form $m_{\mathcal{U}}^{2} O_{\mathcal{U}}^{2}$ with $m_{\mathcal{U}}^{2}$ given by $m_{\mathcal{U}}^{2} \approx\left(\left(\lambda \Lambda^{-d_{\mathcal{U}}}\right)^{2} / 16 \pi^{2}\right) \Lambda_{\text {cut }}^{2}$. Here $\Lambda_{\text {cut }}$ is a cut-off scale of the effective theory. If the cut-off scale is large, the breaking of scale invariance can be large. This situation is similar to the hierarchy problem of Higgs mass. One can eliminate such large loop correction while maintaining the low energy effect of the unparticle and stabilize the theory by making the whole theory supersymmetric.

\footnotetext{
* Corresponding author.

E-mail address: desh@uoregon.edu (N.G. Deshpande).
} 
Supersymmetric theories have many appealing features, they provide a natural solution to the hierarchy problem. With R-parity they provide a natural candidate for dark matter. In the minimal supersymmetric extension of the Standard Model (MSSM), the lightest Higgs boson mass is constrained to be less than about $140 \mathrm{GeV}$. We anticipate the discovery of the Higgs boson at the LHC. A mass in the range of $140 \mathrm{GeV}$ is considered to be consistent with the MSSM. If a light Higgs boson is not found, modifications are needed. We find that the supersymmetrized unparticle effects can relax the Higgs mass limit. This motivates us to consider unparticle effects in a supersymmetric theory, and we therefore propose a model for supersymmetrized version of unparticle interaction.

The model is a minimal extension to MSSM. Besides the usual MSSM contents with R-parity, we add a complex SM singlet chiral unparticle operator which has a scalar unparticle $O_{\mathcal{U}}$ with dimension $d_{\mathcal{U}}$ and also a spinoral partner $\tilde{O}_{\mathcal{U}}$ with dimension $d_{\mathcal{U}}+1 / 2$. The associated F-term $F_{\mathcal{U}}$ has dimension $d_{\mathcal{U}}+1$. Normalizing the supersymmetric unparticle operator to a dimension one chiral field, we write super-field $O s$ as

$$
O s=\left(O_{s \mathcal{U}}+\theta \tilde{O}_{s \mathcal{U}}+\theta^{2} F_{s \mathcal{U}}\right)
$$

where $\left(O_{s \mathcal{U}}, \tilde{O}_{s \mathcal{U}}, F_{s \mathcal{U}}\right)=\Lambda^{1-d_{\mathcal{U}}}\left(O_{\mathcal{U}}, \tilde{O}_{\mathcal{U}}, F_{\mathcal{U}}\right)$. The component super-fields can then be treated in a similar way to the components of usual chiral fields, in constructing the supersymmetric Lagrangian.

Since the unparticle does not have gauge interaction, its interactions with the MSSM particles arise from the super-potential. The lowest dimension operator involving the unparticle is

$$
L_{O}=\lambda \mathrm{H}_{1} \mathrm{H}_{2} \mathrm{Os},
$$

where $H_{1,2}$ are the two Higgs doublets in the MSSM. The component fields and the vacuum expectation values (vev's) are, $H_{1}^{T}=$ $\left(h_{1}^{+},\left(v_{1}+h_{1}^{0}+i a_{1}\right) / \sqrt{2}\right)$, and $H_{2}^{T}=\left(\left(v_{2}+h_{2}^{0}+i a_{2}\right) / \sqrt{2}, h_{2}^{-}\right)$. Depending on the dimension of $O_{\mathcal{U}}$, the theory may or may not be renormalizable. Since the theory is an effective theory, we do not require renormalizability. More complicated operators can also be introduced. In this Letter, we will concentrate on the effects of this simplest operator.

With this operator, the Higgs potential is given by

$$
\begin{aligned}
& V_{\text {SUSY }}=\frac{1}{8}\left(g_{1}^{2}+g_{2}^{2}\right)\left(\left|H_{1}\right|^{2}-\left|H_{2}\right|^{2}\right)^{2}+\frac{1}{2} g_{2}^{2}\left(H_{1}^{\dagger} H_{2}\right)\left(H_{2}^{\dagger} H_{1}\right)+\lambda^{2}\left|H_{1} H_{2}\right|^{2}, \\
& V_{\text {soft }}=\mu_{1}^{2} H_{1}^{\dagger} H_{1}+\mu_{2}^{2} H_{2}^{\dagger} H_{2}-\mu_{12}^{2}\left(H_{1} H_{2}+\text { h.c. }\right) .
\end{aligned}
$$

The term $\lambda^{2}\left|H_{1} H_{2}\right|^{2}$ are obtained by extracting the F-term from the super-potential $L_{O}$.

Note that the operator $O_{\mathcal{U}}$ is similar to the singlet in the next-MSSM (NMSSM) in gauge properties [5]. However, the unparticle does not play the role of a massive particle, terms of the form $O_{\mathcal{U}}^{2}$ are not interpreted as mass terms and such terms are therefore not included in the Higgs potential for Higgs mass calculations. Because Eq. (3) describes an effective theory, $\lambda$ is not constrained to be small as in the NMSSM. The Higgs sector here is very similar to that in $\lambda$ MSSM discussed in [8]. Expanding the potential given above, we obtain the Higgs mass matrices. At the tree level the charged and the pseudoscalar Higgs boson masses $m_{h^{ \pm}}^{2}$ and $m_{A}^{2}$ are

$$
m_{A}^{2}=\mu_{12}^{2} \frac{v_{1}^{2}+v_{2}^{2}}{v_{1} v_{2}}, \quad m_{h^{ \pm}}^{2}=\frac{1}{4}\left(g_{2}^{2}-2 \lambda^{2}+4 \frac{\mu_{12}^{2}}{v_{1} v_{2}}\right)\left(v_{1}^{2}+v_{2}^{2}\right)=m_{W}^{2}\left(1-2 \frac{\lambda^{2}}{g_{2}^{2}}\right)+m_{A}^{2},
$$

where $g_{2}$ is the SM $S U(2)_{L}$ coupling. The neutral Higgs boson mass matrix $M_{h}^{2}$ is modified by a term proportional to $\lambda^{2}$. In the bases $\left(h_{1}^{0}, h_{2}^{0}\right)$, we have

$$
M_{h}^{2}=\left(\begin{array}{cc}
m_{Z}^{2} \cos ^{2} \beta+m_{A}^{2} \sin ^{2} \beta & -\left(m_{Z}^{2}+m_{A}^{2}-\lambda^{2} v^{2}\right) \sin \beta \cos \beta \\
-\left(m_{Z}^{2}+m_{A}^{2}-\lambda^{2} v^{2}\right) \sin \beta \cos \beta & m_{Z}^{2} \sin ^{2} \beta+m_{A}^{2} \cos ^{2} \beta
\end{array}\right),
$$

where $v^{2}=v_{1}^{2}+v_{2}^{2}$ and $\tan \beta=v_{2} / v_{1}$.

We note that the pseudoscalar Higgs mass in this model is the same as that in MSSM. The charged Higgs mass squared is reduced by an amount of $2 \lambda^{2} m_{W}^{2} / g_{2}^{2}$. In this model, at the one loop level if small contributions from unparticle loop effects are neglected, the leading radiative correction to the lightest Higgs boson mass is the same as that in the MSSM. The main effect is to add [7]

$$
\delta=\frac{1}{\sin ^{2} \beta} \frac{3 G_{F} m_{t}^{4}}{\sqrt{2} \pi^{2}} \ln \frac{m_{\tilde{t}}^{2}}{m_{t}^{2}}
$$

to the $(2,2)$ component of the neutral Higgs mass matrix, where $G_{F}$ is the Fermi constant, $m_{t}$ the top quark mass, and $m_{\tilde{t}}^{2}$ the top squark mass. 


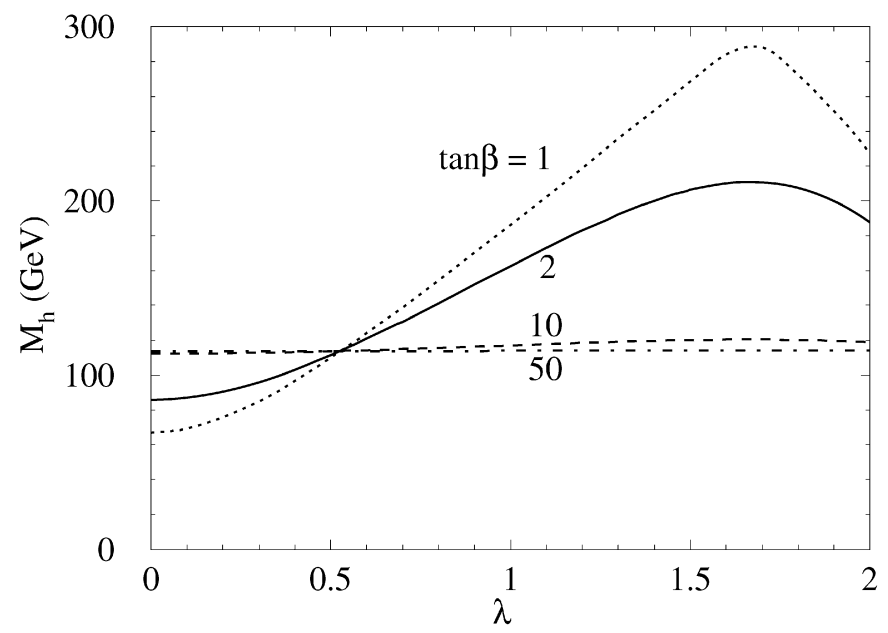

Fig. 1. The lightest neutral Higgs boson mass as a function of $\lambda$ for $\tan \beta=1,2,10$ and 50. The one-loop radiative correction of top quark is included and $m_{A}=400 \mathrm{GeV}$ and $m_{\tilde{t}}=500 \mathrm{GeV}$ is assumed.

The neutral Higgs mass eigenvalues are now given by

$$
\begin{aligned}
m_{h, H}^{2}= & \frac{1}{2}\left\{m_{Z}^{2}+m_{A}^{2}+\delta \mp\left[\left(m_{A}^{2}+m_{Z}^{2}-\lambda^{2} v^{2}\right)^{2}-4\left(m_{A}^{2}-\frac{\lambda^{2} v^{2}}{2}\right)\left(m_{Z}^{2}-\frac{\lambda^{2} v^{2}}{2}\right) \cos ^{2} 2 \beta\right.\right. \\
& \left.\left.-2 \delta\left(m_{Z}^{2}-m_{A}^{2}\right) \cos 2 \beta+\delta^{2}\right]^{\frac{1}{2}}\right\} .
\end{aligned}
$$

We note that the above equation yields same result for the upper bound on $m_{h}$ in the NMSSM. In particular, for fixed $\lambda$ and $m_{A} \rightarrow \infty$, one obtains the overall bound [6],

$$
m_{h}^{2}<m_{Z}^{2} \cos ^{2} 2 \beta+\frac{1}{2} \lambda^{2} v^{2} \sin ^{2} 2 \beta+\delta \sin ^{2} \beta
$$

In our case, we do not restrict $\lambda$ to be necessarily small, or $m_{A}$ to be very large. After this relaxation, we find from Eq. (8) that for a given $m_{A}, m_{h}$ is maximized when $\lambda^{2}=\left(m_{A}^{2}+m_{Z}^{2}\right) / v^{2}$. The maximum value is

$$
m_{h}^{2}=m_{Z}^{2} \sin ^{2} \beta+m_{A}^{2} \cos ^{2} \beta=-m_{Z}^{2} \cos 2 \beta+\lambda^{2} v^{2} \cos ^{2} \beta,
$$

for $\tan \beta \geqslant 1$, and

$$
m_{h}^{2}=m_{Z}^{2} \cos ^{2} \beta+m_{A}^{2} \sin ^{2} \beta=m_{Z}^{2} \cos 2 \beta+\lambda^{2} v^{2} \sin ^{2} \beta,
$$

for $\tan \beta<1$. This result is obvious from the $2 \times 2$ Higgs mass matrix. The highest values of $m_{h}$ is realized when the off-diagonal element goes to zero, i.e. $\lambda^{2}=\left(m_{A}^{2}+m_{Z}^{2}\right) / v^{2}$. The maximum then equals the smaller of the diagonal elements. Since $m_{A}$ is not restricted in SUSY, the value of $m_{h}$ can be large as $m_{A}$ increases. However, Eq. (10) requires that $\lambda$ be correspondingly large. A very large $\lambda$ would not be acceptable because radiative effects induced might be too large. We assume that $\lambda$ of the order unity would be acceptable. The bound in Eq. (9) is realized for fixed value of $\lambda$ and taking the $m_{A} \rightarrow \infty$ limit. For fixed value of $m_{A}$, the limit in Eq. (10) is more stringent than that in Eq. (9). Thus, for a given finite $m_{A}$, the Higgs mass limit comes from Eq. (10) rather than Eq. (9).

In Fig. 1, we plot the lightest scalar Higgs boson as a function of $\lambda$ for different values $\tan \beta$. We take into account only the radiative corrections due to the top and top-squark at one-loop level, the mass values will increase with the inclusion of higher order corrections. For purpose of illustration, we assume that $m_{A}=400 \mathrm{GeV}$ and $m_{\tilde{t}}=500 \mathrm{GeV}$. The dependence of the top-squark mass is logarithmic. It is evident that the $\lambda$ dependence is greatest for low value of $\tan \beta$, becoming less sensitive as $\tan \beta$ increases. For example, at $\lambda \sim 1.7$ the Higgs mass shift due to $\lambda$ for $\tan \beta=2$ is about $120 \mathrm{GeV}$. The shift for $\tan \beta=50$ is less than $1 \mathrm{GeV}$ for all values of $\lambda$. Note that if we had taken a larger $m_{A}$, the peak in Higgs mass would shift to a higher value, and the corresponding value of $\lambda$ would be higher. Therefore, if the Higgs boson mass turns out to be large, the supersymmetric unparticle could be one possible explanation.

Most of the MSSM interactions will not be affected if the fields do not couple directly to the Higgs fields. The SM gauge interactions are not affected. However the new term $L_{O}$ in the super-potential brings some interesting consequences. With R-parity, the lightest supersymmetric particle (LSP) in MSSM is stable and can be a dark matter candidate. In this model the new operator introduces several unparticle interactions which may lead to unstable LSP. We now discuss this issue in some details. 
The new term $L_{O}$ in the super-potential introduces several new supersymmetric unparticle interactions with MSSM particles. We have

$$
L_{O_{\mathcal{U}}}=\left|\mu H_{2}+\lambda \Lambda_{U}^{1-d_{u}} H_{1} O_{u}\right|^{2}+\left|\mu H_{1}+\lambda \Lambda_{U}^{1-d_{u}} H_{2} O_{u}\right|^{2}+\lambda \Lambda_{\mathcal{U}}^{1-d_{\mathcal{U}}}\left(\tilde{H}_{1} \tilde{H}_{2} O_{\mathcal{U}}+H_{1} \tilde{H}_{2} \tilde{O}_{\mathcal{U}}+\tilde{H}_{1} H_{2} \tilde{O}_{\mathcal{U}}\right)
$$

where the field (operator) with "tilde" indicates the suer-partner field (operator).

A neutral Higgsino can decay into an spinor unparticle due to terms $H_{1} \tilde{H}_{2} \tilde{O}_{\mathcal{U}}+\tilde{H}_{1} H_{2} \tilde{O}_{\mathcal{U}}$. After the Higgs doublets develop nonzero vev's, a matrix element, $M\left(\tilde{H}_{i} \rightarrow \tilde{\mathcal{U}}\right)=\lambda \Lambda_{\mathcal{U}}^{1-d_{\mathcal{U}}} v_{j} \tilde{H}_{i} \tilde{O}_{\mathcal{U}}$, is generated. Here $i$ and $j$ take the values 1 and 2 with $i \neq j$. If the scale invariance of unparticle is unbroken down to very low energy, the phase space for the unparticle is proportional $\theta\left(p^{0}\right) \theta\left(p^{2}\right)$, where $p$ is the momentum of the unparticle and $\theta$ is the step-function. This property allows the Higgsino of any mass to decay into an unparticle with

$$
\Gamma\left(\tilde{H}_{i} \rightarrow \tilde{\mathcal{U}}\right)=\left|\lambda \Lambda_{\mathcal{U}}^{1-d_{\mathcal{U}}} v_{j}\right|^{2} \frac{m_{\tilde{H}_{i}}}{2} A_{d_{\mathcal{U}}}\left(m_{\tilde{H}_{i}}^{2}\right)^{d_{\mathcal{U}}-2} \theta\left(m_{\tilde{H}}\right) \theta\left(m_{\tilde{H}}^{2}\right),
$$

where $A_{d_{\mathcal{U}}}=\left(16 \pi^{5 / 2} /(2 \pi)^{2 d_{\mathcal{U}}}\right) \Gamma\left(d_{\mathcal{U}}+1 / 2\right) /\left(\Gamma\left(d_{\mathcal{U}}-1\right) \Gamma\left(2 d_{\mathcal{U}}\right)\right)$.

It is clear that if the LSP in the MSSM has finite mixing with Higgsino, it will not be stable in this model. This makes the conventional explanation of LSP as dark matter candidate untenable. A possible solution to maintain all usual weakly interacting massive particle (WIMP) MSSM dark matter is that the LSP contains no Higgsino component. This requires fine tuning and is not natural. Here we point out that the new unparticle interactions introduced in $L_{O}$ solves the problem and maintain the LSP to be the usual WIMP dark matter by itself in a natural way.

The crucial point for this solution is that some of the new interactions, after the Higgs doublets develop vev's, break scale invariance explicitly, such as $v_{i}^{2} O_{\mathcal{U}}^{2}$ from $\left|H_{i} O_{\mathcal{U}}\right|^{2}$ term. One may also introduce a SUSY breaking terms, $\mu_{\operatorname{SUSY}} \lambda \Lambda^{1-d_{\mathcal{U}}} H_{1} H_{2} O_{\mathcal{U}}$ and $H_{i}^{\dagger} H_{i} O_{\mathcal{U}}$ in the theory. These terms induce terms of the form $v_{i} v_{j} O_{\mathcal{U}}$ which also breaks the scale invariance. Some implications for such an operator has been discussed in Ref. [3]. Assuming that the scale for these scale invariant breaking effects is $\mu^{2}$, it was suggested in Ref. [3] that the phase space should be changed to be proportional to $\theta\left(p^{0}\right) \theta\left(p^{2}-\mu^{2}\right)$. This implies that the Higgsino cannot decay into an unparticle if its mass is less than $\mu$ and would be stable. The relevant scale is proportional to $v_{i} v_{j}$ and is around the electroweak scale. If this is indeed the case, the LSP in MSSM can still be a good candidate for WIMP dark matter. On the other hand, if $\mu$ is less than the mass of the LSP, the LSP would decay into the lightest supersymmetric unparticle state and this could be a dark matter candidate. The properties of dark matter will be modified, because the lightest supersymmetric unparticle state has even smaller interaction with ordinary matter.

The new interactions due to $L_{O}$ can also change Higgs boson decay properties, in particular increasing the invisible decay rate for Higgs bosons. For example, the supersymmetric breaking A-term, $\mu_{\operatorname{SuSY}} \lambda \Lambda^{1-d_{\mathcal{U}}} \mathrm{H}_{1} \mathrm{H}_{2} \mathrm{O}_{\mathcal{U}}$ can induce a term $\mu_{\operatorname{SUSY}} \lambda \Lambda^{1-d_{\mathcal{U}}}\left(v_{i} / \sqrt{2} h_{j}^{0}\right) O_{\mathcal{U}}$ leading to Higgs decay into an unparticle if the Higgs bosom mass is larger than $\mu$. The decay width is given by

$$
\Gamma(h \rightarrow \mathcal{U})=\frac{\left|\mu_{\operatorname{SUSY}} \lambda \Lambda^{1-d_{\mathcal{U}}}\right|^{2}}{2 m_{h}}\left|\frac{v \sin (\alpha+\beta)}{\sqrt{2}}\right|^{2} A_{d_{\mathcal{U}}}\left(m_{h}^{2}\right)^{d_{\mathcal{U}}-2} \theta\left(m_{h}\right) \theta\left(m_{h}^{2}-\mu^{2}\right),
$$

where $\alpha$ is the mixing angle for the neutral Higgs mixing with $h=\cos \alpha h_{1}^{0}+\sin \alpha h_{2}^{0}$ and $H=-\sin \alpha h_{1}^{0}+\cos \alpha h_{2}^{0}$. One can obtain the decay rate for $H$ by replacing $\sin (\alpha+\beta)$ by $\cos (\alpha+\beta)$ in the above expression.

The term $\lambda^{2} \Lambda_{\mathcal{U}}^{2-2 d_{\mathcal{U}}} v_{i} h_{i}^{0} O_{\mathcal{U}}^{2} / 2$ induced from $\left|H_{i} O_{\mathcal{U}}\right|^{2}$, will cause Higgs $h_{i}^{0}$ to decay into two unparticles if the Higgs boson mass is larger than $2 \mu$. These decays will contribute to the invisible decay width of Higgs particle, and affect Higgs search at LHC and ILC. We will present our detailed analysis elsewhere.

In summary we have proposed an unparticle supersymmetric model by introducing an supersymmetric unparticle operator to the MSSM. We introduced the lowest order operator in the super-potential. This operator helps to relax the upper limit on the lightest Higgs boson mass. This operator also introduces several unparticle interactions in the theory leading to interesting phenomenology. In particular, a neutral Higgsino can decay into an spinor unparticle. If the LSP in the MSSM has finite mixing with Higgsino, it will not be stable in this theory and it cannot be a dark matter candidate. However, the same operator also induces scale invariant violation at the electroweak scale. If this scale is larger than the LSP mass, the LSP will again be stable and be a valid dark matter candidate. The new interactions can also induce new invisible decay channels and affect Higgs search at LHC and ILC.

\section{Note added}

While we were finishing our paper, Ref. [9] appeared which also considered some supersymmetric aspects of unparticle effects.

\section{Acknowledgements}

This work was supported in part Grants No. DE-FG02-96ER40949 of the US Department of Energy, and NSC and NCTS of ROC. 


\section{References}

[1] H. Georgi, Phys. Rev. Lett. 98 (2007) 221601, hep-ph/0703260.

[2] H. Georgi, arXiv: 0704.2457 [hep-ph];

K. Cheung, W.Y. Keung, T.C. Yuan, arXiv: 0704.2588 [hep-ph];

M. Luo, G. Zhu, arXiv: 0704.3532 [hep-ph];

C.H. Chen, C.Q. Geng, arXiv: 0705.0689 [hep-ph];

G.J. Ding, M.L. Yan, arXiv: 0705.0794 [hep-ph];

Y. Liao, arXiv: 0705.0837 [hep-ph];

T.M. Aliev, A.S. Cornell, N. Gaur, arXiv: 0705.1326 [hep-ph];

X.Q. Li, Z.T. Wei, arXiv: 0705.1821 [hep-ph];

C.D. Lu, W. Wang, Y.M. Wang, arXiv: 0705.2909 [hep-ph];

M.A. Stephanov, arXiv: 0705.3049 [hep-ph];

N. Greiner, arXiv: 0705.3518 [hep-ph];

H. Davoudiasl, arXiv: 0705.3636 [hep-ph];

D. Choudhury, D.K. Ghosh, Mamta, arXiv: 0705.3637 [hep-ph];

T.M. Aliev, A.S. Cornell, N. Gaur, arXiv: 0705.4542 [hep-ph];

P. Mathews, V. Ravindran, arXiv: 0705.4599 [hep-ph];

S. Zhou, arXiv: 0706.0302 [hep-ph];

G.J. Ding, M.L. Yan, arXiv: 0706.0325 [hep-ph];

C.H. Chen, C.Q. Geng, arXiv: 0706.0850 [hep-ph];

Y. Liao, J.Y. Liu, arXiv: 0706.1284 [hep-ph];

M. Bander, J.L. Feng, A. Rajaraman, Y. Shirman, arXiv: 0706.2677 [hep-ph];

T.G. Rizzo, arXiv: 0706.3025 [hep-ph];

K. Cheung, W.Y. Keung, T.C. Yuan, arXiv: 0706.3155 [hep-ph];

H. Goldberg, P. Nath, arXiv: 0706.3898 [hep-ph];

S.L. Chen, X.G. He, H.C. Tsai, arXiv: 0707.0187 [hep-ph];

R. Zwicky, arXiv: 0707.0677 [hep-ph];

T. Kikuchi, N. Okada, arXiv: 0707.0893 [hep-ph];

R. Mohanta, A.K. Giri, arXiv: 0707.1234 [hep-ph];

C.S. Huang, X.H. Wu, arXiv: 0707.1268 [hep-ph];

A. Lenz, arXiv: 0707.1535 [hep-ph];

D. Choudhury, D.K. Ghosh, arXiv: 0707.2074 [hep-ph];

X.-Q. Li, Y. Liu, Z.-T. Wei, arXiv: 0707.2285 [hep-ph].

[3] P.J. Fox, A. Rajaraman, Y. Shirman, arXiv: 0705.3092 [hep-ph].

[4] S.L. Chen, X.G. He, arXiv: 0705.3946 [hep-ph].

[5] H.P. Nilles, M. Srednicki, D. Wyler, Phys. Lett. B 120 (1983) 346;

J.M. Frere, D.R.T. Jones, S. Raby, Nucl. Phys. B 222 (1983) 11;

J.P. Derendinger, C.A. Savoy, Nucl. Phys. B 237 (1984) 307;

J.R. Ellis, J.F. Gunion, H.E. Haber, L. Roszkowski, F. Zwirner, Phys. Rev. D 39 (1989) 844.

[6] M. Drees, Int. J. Mod. Phys. A 4 (1989) 3635.

[7] M. Drees, R. Godbole, P. Roy, Theory and Phenomenology of Sparticles, World Scientific, Hackensack, USA, 2004, 555 p.;

H. Baer, X. Tata, Weak Scale Supersymmetry, Cambridge Univ. Press, Cambridge, UK, 2006, 537 p.

[8] R. Barbieri, L.J. Hall, Y. Nomura, V.S. Rychkov, Phys. Rev. D 75 (2007) 035007, hep-ph/0607332.

[9] H. Zhang, C.S. Li, Z. Li, arXiv: 0707.2132 [hep-ph];

Y. Nakayama, arXiv: 0707.2451 [hep-ph]. 